\title{
[gw22-e0160] IMPROVEMENT OF INFARCTED HEART BY THE TRANSPLANTATION OF ADIPOSE-DERIVED STEM CELLS WITH INJECTABLE TEMPERATURE- RESPONSIVE CHITOSAN HYDROGEL
}

Junjie Yang ${ }^{1}$, Zhiqiang Liu², Zhenliang Yuan², Yundai Chen', Changyong Wang ${ }^{1}$ Academy Of Military Medical Sciences, Beijing, China; ${ }^{2}$ Pla General Hospital, Beijing, China

\subsection{6/heartjnl-2011-300867.35}

Objective Stem cell-based therapy can preserve cardiac function but is limited by the low cell retention within ischemic tissues. Injectable tissue engineering materials are well known for maintain cell survival and enhance cell-based therapies' efficacy. We aimed to studying the usefulness of the combination of Injectable temperature-responsive chitosan hydrogel (as scaffold) and adipose-derived stem cells (ADSCs) to treat myocardial infarction.

Methods Rat ADSCs were isolated from subcutaneous adipose tissues and further confirmed by its surface phenotype as well as multi-potential differentiation. Temperature-responsive chitosan hydrogel (chitosan), made by specific technique, was then co-injected with ADSCs into the left ventricular wall of rat myocardial infarction models. The structure and functional consequences of transplantation were determined by detailed histological analysis and echocardiography.

Results Most cultured ADSCs expressed CD 29 and CD90, and negative for CD34 and CD45. After injection, both the 48 -cell retention and 4-week graft size were significantly higher and larger in the chitosan+ADSCs group than those of the ADSCs group alone $(\mathrm{p}<0.01)$. 4 weeks after transplantation, the heart function, as well as the arteriole densities in the infarcted area, improved significantly in the chitosan+ADSCs group compared with that of the ADSCs group $(p<0.01)$.

Conclusion The method of in situ injectable tissue engineering combining chitosan hydrogel with ADSCs is promising clinically for preserving the function of infarcted hearts. 